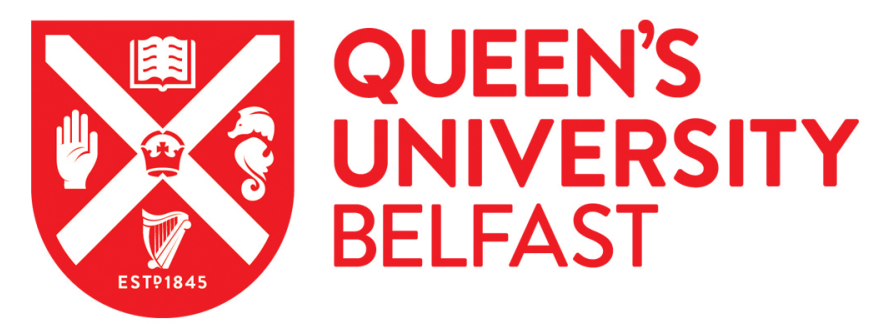

\title{
The cost-effectiveness of alternative vision screening models among preschool children in rural China
}

Wang, L., Congdon, N., Hogg, R. E., Zhang, S., Li, M., Shi, Y., Jin, L., He, F., Wang, H., Boswell, M., \& Iyer, M. (2018). The cost-effectiveness of alternative vision screening models among preschool children in rural China. Acta Ophthalmologica. https://doi.org/10.1111/aos.13954

Published in:

Acta Ophthalmologica

Document Version:

Peer reviewed version

Queen's University Belfast - Research Portal:

Link to publication record in Queen's University Belfast Research Portal

Publisher rights

(c) 2018 Acta Ophthalmologica Scandinavica Foundation. Published by John Wiley \& Sons Ltd. This work is made available online in accordance with the publisher's policies. Please refer to any applicable terms of use of the publisher.

\section{General rights}

Copyright for the publications made accessible via the Queen's University Belfast Research Portal is retained by the author(s) and / or other copyright owners and it is a condition of accessing these publications that users recognise and abide by the legal requirements associated with these rights.

Take down policy

The Research Portal is Queen's institutional repository that provides access to Queen's research output. Every effort has been made to ensure that content in the Research Portal does not infringe any person's rights, or applicable UK laws. If you discover content in the Research Portal that you believe breaches copyright or violates any law, please contact openaccess@qub.ac.uk. 


\section{The cost-effectiveness of alternative vision screening models among preschool}

children in rural China

Lei Wang, ${ }^{1,2}$ Nathan Congdon, ${ }^{3,4,5}$ Ruth E. Hogg, ${ }^{3,4,5}$ Siqi Zhang, ${ }^{2}$ Mengjie Li, ${ }^{1,2}$ Yaojiang Shi, ${ }^{2}$ Ling Jin, ${ }^{4}$ Fei He, ${ }^{6}$ Huan Wang, ${ }^{2}$ Matthew Boswell, ${ }^{7}$ Mony Iyer ${ }^{8}$

1 International Business School, Shaanxi Normal University, Shaanxi China; Center for Experimental Economics of Education, Shaanxi Normal University, Shaanxi China;

2 Center for Experimental Economics of Education, Shaanxi Normal University, Shaanxi China;

3 Centre for Public Health, Queen's University Belfast, Belfast, UK

4 State Key Laboratory of Ophthalmology, Zhongshan Ophthalmic Center, Sun Yat-sen University, Guangzhou, China

5 Orbis International, New York City, New York, USA

6 Food and Resource Economics Department, University of Florida, Gainesville, FL, USA

7 Freeman Spogli Institute of International Studies, Stanford University, Stanford, California, USA

8 OneSight, Mason, Ohio, USA

Address for correspondence: Prof Shi Yaojiang, Center for Experimental Economics in Education (CEEE), Shaanxi Normal University West Chang'an Road No. 620, Chang'an District, Xi'an, 710119, China

Telephone number/Fax: (86)2981530872

Funding source for statement: Supported by the 111 Project (Grant number B16031). Prof Congdon is supported by the Ulverscroft Foundation and the Thousand Man Plan of the government of China.

Conflicts of interest: We declare that we have no conflicts of interest.

Length: Text: 3356 words, 5 tables, 2 figures, 18 references. Abstract: 250 words

Version: 22 June 2018 


\begin{abstract}
Purpose: To explore the accuracy and cost-effectiveness of three vision screening models among preschool children in rural China.
\end{abstract}

Methods: Vision screening was carried out among children aged 4-5 years in 65 preschools in two counties in Northwest China, using Crowded Single Lea Symbols to test visual acuity. Children were assigned randomly by school to one of three screening models: screening by teachers ( 15 schools, 1,835 children), local optometrists (30 schools, 1,718 children) or volunteers (20 schools, 2,183 children). Children identifying $\geq 2$ symbols incorrectly in either eye failed screening. Accuracy of screening was compared with screenings executed by experienced optometrists among 141 children selected randomly from the three screening models. Direct and indirect costs for each model were assessed. Costs to detect a true case failed screening were estimated.

Results: The sensitivity for three models ranged from $76.9 \%$ to $87.5 \%$, specificity from $84.9 \%$ to $86.7 \%$, and standardized positive predictive value from $83.7 \%$ to $85.7 \%$. None differed significantly between models. The costs per case detected were $\$ 37.53, \$ 59.14$ and $\$ 52.19$ for the teachers, local optometrists and volunteers. In producing the cost estimates for teacher screening and local optometrist screening models, we used a salary 
payment that was identical for both models (with the salary being equal to that of the optometrist). The teacher screening model was the most cost-effective.

Conclusion: Accuracy of screening by teachers, local optometrists and volunteers were the same in this setting, but the use of teachers was most cost-effective, reducing the cost per case detected by almost $40 \%$.

Key words: cost-effectiveness - preschool - rural China - vision screening 


\section{Introduction}

Poor vision is the most common impairment affecting school-aged children in low- and middle-income countries (LMICs), accounting for $48 \%$ of all disability among children aged 5-9 years in the India census of 2001 (Dimension of Disability in India, 2013). The leading and most easily treated cause of visual impairment (visual acuity $<6 / 18$ ) among children is refractive error, affecting 12.8 million children between 5 and 15 years, half of whom live in China (Resnikoff et al. 2008). Spectacles provide a safe and inexpensive treatment and are shown to improve children's educational outcomes (Ma et al. 2014). The first step in providing refractive services to children is vision screening to identify those most likely to be affected.

In developed countries, formal screening programs may be mandated and funded. The American Academy of Pediatrics recommends that vision screening begins at birth and continues throughout a child's regular medical check-ups (American Academy of Pediatrics Committee on Practice and Ambulatory Medicine Section on Ophthalmology, 1996). In the United States, most states require a school vision examination. For years, officials in European countries, including Sweden, Germany and Denmark, have also mandated and funded preschool physician-administered vision screening programmes (Kvarnström et al. 2001; Gandjour et al. 2003; Høeg T et al. 2015).

Vision screening is particularly important in developing countries, where it may be the only timely way to identify childhood vision disorders such as refractive error; 
however, screening in LMICs may only occur informally, if at all. Because trained optometrists are few, insurance coverage is lacking, and parents and teachers fail to detect symptoms, few children in developing settings have access to vision screening (Schimiti et al. 2001). Still, studies have shown that in countries such as India, Iran, and Brazil, optometrist-trained teachers can conduct cost-effective screenings (Limburg et al. 1994; Schimiti et al. 2001; Khandekar et al. 2009).

China has no officially sanctioned national preschool vision screening programme. Practical, effective strategies for early detection are needed. This paper's goal was to explore the accuracy (sensitivity, specificity, and standardized positive predictive value) compared to an experienced, tertiary optometrist and cost effectiveness of three different preschool vision screening methods: screening by local, rural optometrists; by preschool teachers trained by local optometrists; and by lay volunteers.

\section{Methods}

The Institutional Review Board (IRB) at Stanford University fully approved this study's protocol (protocol \#24847). Permission was received from local boards of education and the principals of all participating schools. Since in China school principals are children's legal guardians, and the study involved no intervention beyond screening, the IRB did not require parental consent. Declaration of Helsinki principles were followed throughout. 
Vision screening was carried out among rural preschool children aged 4-5 years in 65 preschools from all townships in Yongshou and Xunyi counties in Xianyang Prefecture, Shaanxi Province, China. Xianyang was randomly selected from among predominantly rural prefectures in Shaanxi, and the two study counties were also randomly selected. Average annual income of rural families was $\$ 1300$ in Yongshou and $\$ 1390$ in Xunyi, compared to Shaanxi's provincial average of $\$ 1300$ reported in the Shaanxi Statistic Yearbook 2016 and the national average of $\$ 1600$ reported in the China Statistic Yearbook 2016. At present, most preschool children in these counties have not undergone vision screening.

For sample selection, we obtained a list of all preschools from each county's education bureau. From this list, we randomly selected 20 rural preschools in Xunyi and 45 in Yongshou. Of these 65 preschools, 17 were public and 48 were private. Our sample included 5736 children. Our sample is representative of China's population. Specifically, the individuals in our sample are all ethnically Han (a group which accounts for more than $90 \%$ of China's population as reported in the China census of 2010), rural-dwelling (more than 50\% of China's population lives in rural areas) and drawn from counties with incomes near the national average (see above).

School surveys were conducted November 2014-April 2015 to determine whether the schools were public or private, how far each school was from the county seat, and whether the school had ever previously implemented a children's vision screening 
program. Enumerators from the research team carried out a census, including name, age and sex of all children.

\section{Vision screening and case definition}

Three vision screening models were used. Two of these (teacher screening and local optometrist screening) were conducted in Yongshou county, the third (volunteer screening) in Xunyi. All preschools in Yongshou were ranked by distance to the county seat, and then, schools were randomly assigned teacher or local optometrist screening.

In the teacher screening model, teachers from the 15 selected schools underwent a half-day training programe implemented by local optometrists and conducted at a central site. Teachers then returned to their own schools and screened a total of 1835 children in their classes in April 2015.

In the local optometrist screening model, a team of three local optometrists based at the county hospital was trained for half a day by experienced optometrists ${ }^{1}$ from a tertiary eye hospital (Zhongshan Ophthalmic Center, Guangzhou, China). This team visited the 30 selected preschools by car, screening 1718 children in November 2014. In the volunteer screening model, 10 student volunteers from Shaanxi Normal University underwent a half day of training by the tertiary optometrists who trained the local optometrists, and then carried out screenings on 2183 children in 20 preschools in

\footnotetext{
1 There was a difference among the optometrists that provided the training in the three groups. While both sets of optometrists were experienced, the optometrists from the tertiary eye hospital (who trained the local optometrists and the volunteers) had formal qualifications; the local optometrists (who trained the teachers) did not have formal qualifications.
} 
March 2015. The volunteer screeners were brought to the schools by vans and stayed in local hotels.

All screeners in each model carried out the same vision screening protocol, using the Crowded Single Lea Symbols Visual Acuity Test (Good-Lite, Inc., Elgin, Il, USA) at $3 \mathrm{~m}$. Separate disc cards for right and left eyes and for 4- and 5-year-olds each had four equal-sized symbols (apple, house, circle and square) surrounded by "crowding” lines, which have been shown to compromise visual performance in amblyopic children. A viewing window allowed only one symbol to be rotated into view at a time. Screeners used a lap card displaying all four symbols to aid children in identifying them, with formal testing only beginning once the child could point correctly to each of the four symbols on the lap card when the symbol appeared in the viewing window on the disc card at a distance of $1 \mathrm{~m}$.

With the child positioned $3 \mathrm{~m}$ from the card, an assistant screener covered the left eye with occluding glasses, while the main screener rotated the wheel on the right eye side on the disc card until the first Lea symbol was visible in the window. The child was then asked to verbally identify or point to the corresponding Lea symbol on the lap card. After each response, the next symbol was presented in the window. If the child identified two or more symbols incorrectly, the eye was considered to have failed screening. If the child correctly identified all the symbols or only got one wrong, successive symbols were tested until the child either successfully identified four symbols (passed) or incorrectly 
identified two symbols on a line of four (failed). This procedure was repeated for the left eye.

\section{Screening Audit}

In June 2015, a team consisting of an experienced optometrist from a tertiary eye hospital (Zhongshan Ophthalmic Center) and a trained volunteer from Shaanxi Normal University carried out an audit of screening accuracy of the three models. Auditors examined children from the 20 preschools that had $\geq 2$ children fail vision screening. From these schools, five children who had passed the screening and three children who had failed were selected at random. In total, auditors examined 141 children. The monitoring optometrist, masked to the original screening results, performed visual acuity screening on all selected children using the above protocol with assistance of the trained volunteer.

\section{Cost effectiveness data collection}

Cost-effectiveness analysis data were collected from the screening as performed by the three sets of screeners. All costs were reported in 2015 US dollars ( 1 renminbi $=$ US\$0.15). During the screening, costs included expenditures for training screeners (Appendix TableA1, row 1) and screening costs (row 2). For the local optometrist model, we included a payment to the optometrist for services rendered as part of the overall cost of screening (US\$26 per day). For the teacher screening model, we assumed that teachers 
received the same level of compensation for screening as the local optometrists (also US\$26 per day). Fixed costs such as tool kits and training materials were excluded.

\section{Statistical Methods}

Student and school characteristics were presented by mean (standard deviation) or median (interquartile range) for continuous variables and frequency (percentage) for categorical variables. To examine potential associations with accuracy of screening in each of the three models, we compared differences in these characteristics between the screening models and also in the proportion of children failing screening under each model. Linear regression was used for continuous variables with normal distribution, median regression was used for the variable of distance (which was not normally distributed), and logistic regression was used for categorical variables. Clustering within school was accounted for in all comparisons in linear regression and logistic regression, and using a bootstrap procedure that allowing for clustering for median regression.

Sensitivity, specificity and standardized positive predictive value (PPV) were calculated separately for each screening model, with tertiary optometrists considered as the gold standard. We adopted the method proposed by Thomas $(2011,2014)$ to compute the "standardized positive predictive value (standardized PPV)," which is designed to reduce prevalence bias when comparing one diagnostic test with another. Standardized PPVs were calculated for each screening model using the following formula: 


$$
\text { Standardized PPV }=\frac{\text { Sensitivity } \times 0.5}{\text { Sensitivity } \times 0.5+(1-\text { Specificity }) \times(1-0.5)}
$$

In order to compare the differences between the three screening models in sensitivity, specificity and the standardized PPV, we also used the chi-square test for pairwise comparisons of area under the receiver operating characteristic (ROC) curves (AUCs) of the three screening models.

All economic evaluations were performed as cost-effectiveness analyses. The outcome measures were true cases of failed vision screening detected based on our sample. The cost-effectiveness of three screening models was compared using the incremental cost effectiveness ratio (ICER) (Phillips \& Thompson, 2003). This ratio is defined as the additional cost of a specific screening model divided by the additional number of true-positive cases of failed vision screening detected compared with the next less-expensive model. Screening models that are less effective and costlier than an alternative, and screening models with a higher cost-effectiveness ratio than a more effective alternative model, are both ruled out. A sensitivity analysis was performed, varying costs upward and downward by $10 \%$ for each of the three screening models.

\section{Results}

Of the 5,736 children who underwent screening, $52.0 \%(\mathrm{n}=2983)$ were boys and the mean (SD) age of the total sample was $5.04(0.80)$ years. Only 3.00\% had previously undergone visual acuity testing, and 30.0\% attended public (as opposed to private) 
preschools (Table 1). A total of 194 (3.38\%) children failed vision screening (Figure 1). As part of the study's protocol, we informed all parents of their children's screening results. In addition, when children failed their screenings, the parents were explicitly told that they should consider taking their children to local hospitals for further eye examination. The prevalence of children failing screening was higher among volunteers $(96 / 2183=4.40 \%)$ compared to local optometrists $(52 / 1718=3.03 \%, \mathrm{p}=0.05)$ and trained teachers $(46 / 1835=2.51 \%, \mathrm{p}=0.02)$. Among all the children, only a total of 141 children underwent secondary testing by the experienced optometrists: $46(32.6 \%)$ in the local optometrist regimen, $39(27.7 \%)$ in the teacher regimen and $56(39.7 \%)$ in the volunteer regimen (Figure 1). 
[Table 1 is supposed to be here]

[Figure 1 is supposed to be here]

Among the 141 children screened by the monitoring optometrist during the quality check, $40(28.4 \%)$ failed the initial screening carried out by the teacher, local optometrist or volunteer, while $30(21.3 \%)$ failed screening by the monitoring optometrist. According to the study's protocol, for those individuals that were identified as false positive (16 out of 141) or false negative (6 out of 141), we referred them to an ophthalmologist for diagnosis and treatment. The overall sensitivity, specificity, and standardized PPV of the initial screening (summing across all three screening models) were $80.0 \%, 85.6 \%$, and $85.1 \%$. For the three screening models, the sensitivities ranged from $76.9 \%$ to $87.5 \%$, the specificities from $84.9 \%$ to $86.7 \%$ and the standardized PPVs from $83.7 \%$ to $85.7 \%$ (Table 2 ). None of these values differed statistically significantly from one another. We also plotted the ROC curves to compare the accuracy between the three different screening models (Figure 2). The p values for the pairwise comparisons of area under the AUCs between the three screening models were all $>0.60$. (Figure 2).

[Table 2 is supposed to be here]

[Figure 2 is supposed to be here]

The average cost per screening ranged from US\$0.61 in the teacher screening model to US\$1.20 in the volunteer screening model, and the costs per true positive case of failed vision screening were US\$37.53, US\$52.19 and US\$59.14 in the teacher, volunteer and 
local optometrist screening models, respectively. Table 3 illustrates that the ICERs for the teacher, local optometrist and volunteer screening models were US\$0.38, US\$1.35 and US $\$ 0.25$, respectively. Thus, the local optometrist screening model was excluded as a more costly and less effective alternative. Having ruled out the local optometrist screening model, ICERs were recalculated for the teacher and volunteer screening models, as shown in Table 4. The volunteer screening model was 'dominated' by the teacher screening model as the latter was more effective and cost less to produce an additional unit of effect (US\$0.38 compared with US\$0.98). The volunteer screening model was therefore excluded as a dominated alternative, and the teacher screening model was thus the most cost effective. In previous cost-effectiveness studies conducted in developing countries, such as Brazil and India (Limburg et al. 1994; Schimiti et al. 2001), the most common cost-effectiveness screening method is to assign screening as a responsibility to teachers (the teacher screening model). The idea is that since screening is a true educational input, it is a teacher's duty to provide it. Because a teacher's regular responsibilities are remunerated by his or her annual salary, in these papers when calculating the cost of screening, the teacher's salary is either not included or it is included as a reduced cost. However, an alternative approach is to do the costeffectiveness analysis using the same salary costs as the local optometrists instead of reduced costs for teachers. The reason for doing this is that if there is going to be screening, the screening should reflect the full opportunity cost under the assumption that 
teachers time should be valued equally with that of optometrists. It is this second alternative that is used in this paper. When costs are calculated in this way, the teacher screening model is the most cost-effective method. A sensitivity analysis performed by varying costs upwards and downwards by $10 \%$ did not affect this conclusion.

[Table 3 is supposed to be here]

[Table 4 is supposed to be here]

\section{Discussion}

We found that the rate of failed vision screening among preschool children in rural China was $3.38 \%$. Even if only a portion of this turns out to represent true vision disorders, such a high prevalence suggests it may be valuable to conduct systematic vision screenings for children at this age (American Academy of Pediatrics Committee on Practice and Ambulatory Medicine Section on Ophthalmology,1996; Kvarnström et al. 2001; Gandjour et al. 2003; Høeg T et al. 2015). Unfortunately, we found that few preschool students had previously been screened for any type of vision disorder. It is important that these children receive treatment, particularly in view of our recent trial demonstrating the educational benefits of improving vision with refraction in school-aged children (Kvarnström et al. 2001), and the fact that amblyopia treatment is typically successful for children in this age group (Weinstein et al. 1996).

We found that the three alternative screening models did not differ significantly in accuracy, though the ICER analysis showed that the teacher screening model was more 
cost-effective than both the local optometrist and volunteer screening models. This finding is consistent with studies in other developing countries, such as Brazil and India, where teachers trained by local optometrists provided cost-effective screening (Limburg et al. 1994; Schimiti et al. 2001; Gandjour et al. 2003). However, these studies did not compare cost-effectiveness of different screening models as our paper did. In India, teachers screened their students without any additional incentives. It was cost-effective since the cost per child was low (US\$0.03) (Limburg et al. 1994). However, in the India study, there was only one intervention and no comparison groups using alternative screening models. In Germany, the most cost-effective approach was to have ophthalmologists screen - with that average cost being US\$36, compared to screenings by orthoptist and pediatrician/general practitioner (Gandjour et al., 2003). Obviously, screenings by ophthalmologists were much more expensive compared to our teacher screening model (US\$0.61).

There are several reasons for this. Because volunteers came from urban areas at some distance from the schools, they needed to be compensated for travel and accommodation. Local optometrists had to travel from the county seat to the sample rural schools and had to be provided with subsidies to conduct screenings at schools (which included time spent organizing the screenings inside the schools). Although we assumed that teachers were compensated for their screening services at the same rate as optometrists, they did not need to travel and needed less time to organize the screening 
since they were familiar with all students in their classes. Both of these advantages led to the lower screening costs for the teacher screening model.

Our study has several strengths. First, to the best of our knowledge, this is the first study of preschool vision screening in rural China. Although there have been studies on such screening in other developing countries, this study is the first to use Lea Symbols, which have been validated as the most accurate method for lay screeners in the Vision In Preschoolers study (Graf and Becker, 1999; Jones et al. 2003; Khandekar et al. 2009). Second, the study design three evaluated different locally relevant vision screening strategies for preschool children, which allowed us to compare their accuracy and costeffectiveness.

There are also limitations to this study. The data were collected from rural areas of one province in western China, and although the sample population was made up of Han individuals living in rural communities with per capita incomes near the national average, ours cannot be considered a nationally representative sample. To enhance the representativeness of the findings, additional work should be done elsewhere in China in the future.

We also did not conduct eye examination, which did not allow us to confirm diagnoses of specific vision disorders. Further, our estimates of the various accuracy parameters (sensitivity, specificity, standardized PPV) are based on expert evaluation of a random $2.46 \%$ sample of children, rather than re-examination of every child. 
From a policy perspective, our results show that the prevalence of failed vision screening in preschool children in rural China is relatively high, implying that a mandatory vision screening programme should be considered. In a resource-limited setting such as rural China, teachers appear to offer the most cost-effective way to carry out such screening. Furthermore, the teacher model we tested is a strong candidate for upscaling. The LEA Symbols test is validated for lay use and simple to understand. It is also easier to co-ordinate teacher-conducted screening, as preschool children recognize and are more likely to co-operate with their teachers than with unfamiliar doctors and volunteers. Currently, 75\% of Chinese children attend pre-schools (Ministry of Education of the People's Republic of China, 2016), and the proportion is rising. This offers a unique opportunity to identify and treat vision disorders in these children before they begin formal schooling, potentially avoiding impediments to optimal educational performance. 


\section{Reference}

American Academy of Pediatrics Committee on Practice and Ambulatory Medicine Section on Ophthalmology (1996): Eye examination and vision screening in infants, children, and young adults. Pediatrics 98: 153-7.

Dimension of Disability in India, chapter 4 (2013): http://mospi.nic.in/Mospi_New/upload/disablity_india_statistical_data_11mar2011 /Chapter\%204-Dimension_Disability.pdf. Table 4.3 (b).

Gandjour A, Schlichtherle S, Neugebauer A, Russmann W, Lautervach K W (2003): A cost-effectiveness model of screening strategies for amblyopia and risk factors and its application in a German setting. Optom Vis Sci 80: 259-69.

Graf M, Becker R (1999): Determining visual acuity with LH symbols and Landolt rings. Klin Monatsbl Augenheilkd 215: 86-90.

Høeg T, Moldow B, Ellervik C, Klemp K, Erngaard D, La Cour M, Buch H. Danish (2015): Rural Eye Study: The association of preschool vision screening with the prevalence of amblyopia. Acta ophthalmol 93: 322-329.

Jones D, Westall C, Averbeck K, Abdolell M (2003): Visual acuity assessment: a comparison of two tests for measuring children's vision. Ophthalmic Physiol Opt 23: $541-6$.

Khandekar R, Parast N, Arabi A (2009): Evaluation of 'vision screening' program for three to six-year-old children in the Republic of Iran. Indian J Ophthalmol 57: 43742. 
Kvarnström G, Jakobsson P, Lennerstrand G (2001): Visual screening of Swedish children: an ophthalmological evaluation. Acta Ophthalmologica 79: 240-4.

Limburg H., Vaidyanathan K, Dalal HP (1994): Cost-effective screening of schoolchildren for refractive errors. World Health Forum16: 173-8.

Ma X, Zhou Z, Yi H et al. (2014): Effect of providing free glasses on children's educational outcomes in China: cluster randomized controlled trial. BMJ 349: g5740.

Ministry of Education of the People's Republic of China (2016): Whole country education the business development statistics official gazette. http://moe.edu.cn/srcsite/A03/s180/moe_633/201607/t20160706_270976.html.

Phillips C, Thompson G (2003): What is cost-effectiveness? Hayward Medical Communications.

Resnikoff S, Pascolini D, Mariotti SP, Pokharel GP (2008): Global magnitude of visual impairment caused by uncorrected refractive errors in 2004. Bull World Health Organ 86: 63-70.

Schimiti RB, Costa VP, Gregui MJF, Kara-José N, Temporini ER (2001): Prevalence of refractive errors and ocular disorders in preschool and school children of Ibiporã-PR, Brazil (1989 to 1996). Arquivos Brasileiros de Oftalmologia 64: 37984.

Thomas FH (2011): Standardizing predictive values in diagnostic imaging research. JMRI 33: 505.

Thomas FH (2014): Standardized predictive values. JMRI 39: 1338. 
Weinstein MC, Siegel JE, Gold MR, Kamlet MS, Russell LB (1996): Costeffectiveness in health and medicine. New York: Oxford University. 


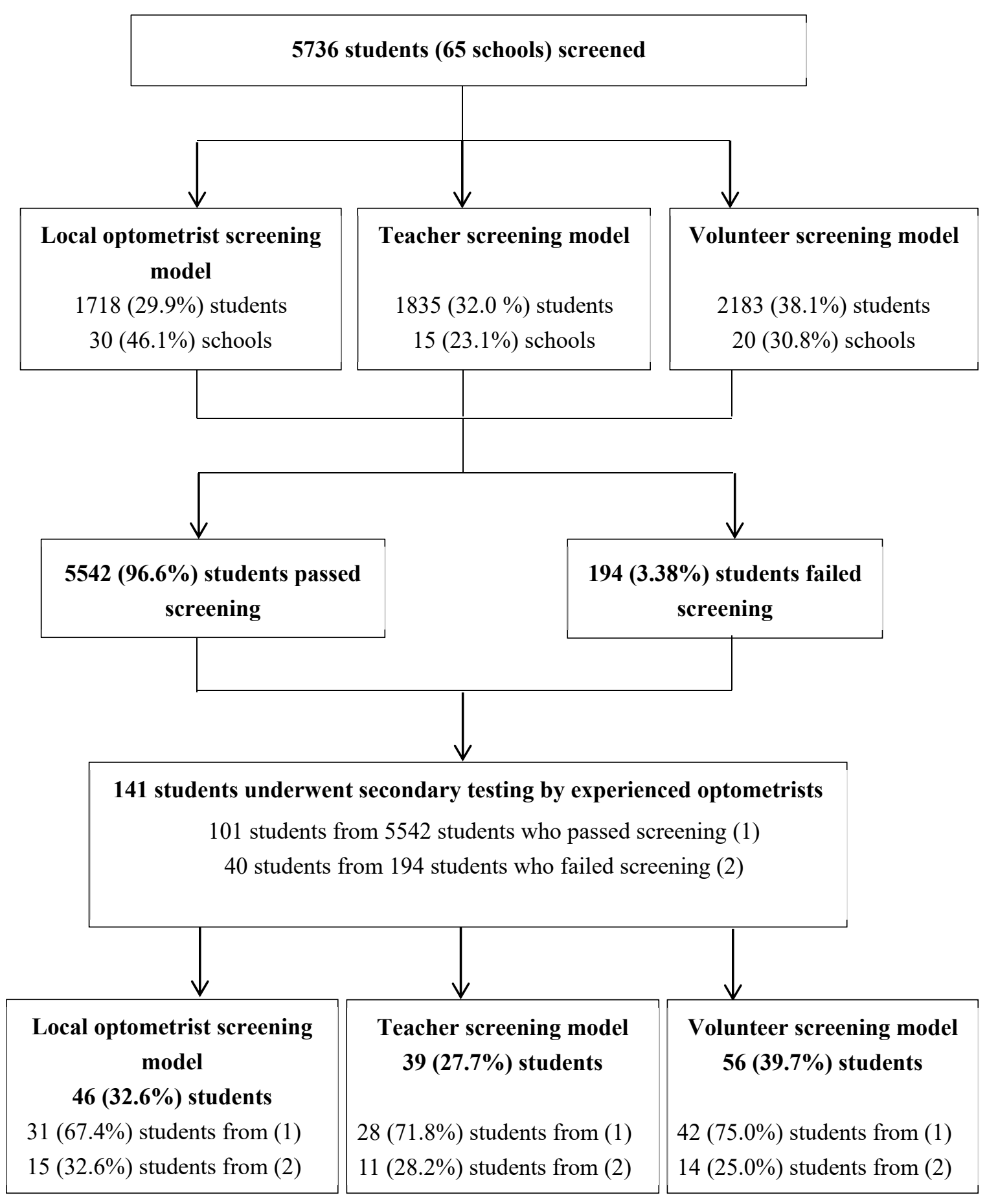

Fig. 1. Flow chart of participants through the study 


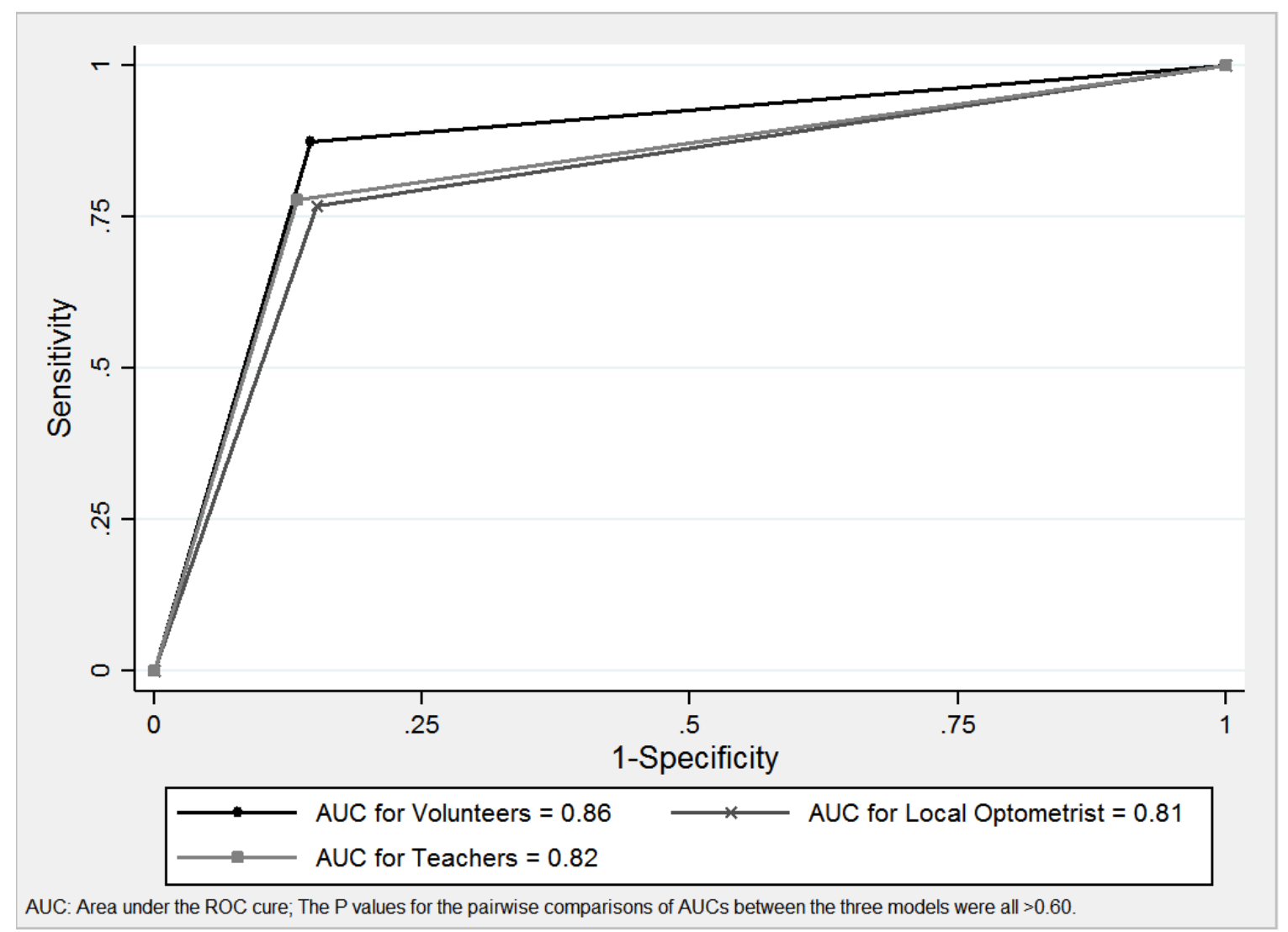

Fig. 2. Receiver operating characteristic (ROC) curve for each screening model in the study. 
Table 1. Baseline characteristics of children by screening model.

\begin{tabular}{|c|c|c|c|c|c|c|c|}
\hline & \multirow[b]{2}{*}{$\begin{array}{c}\text { Total } \\
(n=5736)\end{array}$} & \multirow{2}{*}{$\begin{array}{c}\text { Local } \\
\text { optometrist } \\
\text { screening model } \\
(n=1718 \\
29.9 \%)\end{array}$} & \multirow{2}{*}{$\begin{array}{c}\text { Teacher } \\
\text { screening model } \\
\left(\begin{array}{c}n=1835 \\
32.0 \%)\end{array}\right.\end{array}$} & \multirow[b]{2}{*}{$\begin{array}{c}\text { Volunteer } \\
\text { screening model }(n \\
=2183,38.1 \%)\end{array}$} & \multicolumn{3}{|c|}{ p-value } \\
\hline & & & & & $\begin{array}{c}\text { Local } \\
\text { optometrist } \\
\text { Versus } \\
\text { Teacher }\end{array}$ & $\begin{array}{c}\text { Local } \\
\text { optometrist } \\
\text { versus Volunteer }\end{array}$ & $\begin{array}{c}\text { Teacher } \\
\text { versus } \\
\text { Volunteer }\end{array}$ \\
\hline \multicolumn{8}{|l|}{ Student characteristics } \\
\hline Male, $n(\%)$ & $2,983(52.0 \%)$ & $900(52.4 \%)$ & $947(51.6 \%)$ & $1,136(52.0 \%)$ & $0.642^{*}$ & $0.832^{*}$ & $0.739^{*}$ \\
\hline Age (years) (Mean, SD) & $5.04(0.80)$ & $4.65(0.77)$ & $5.26(0.77)$ & $5.17(0.74)$ & $<0.001^{\dagger}$ & $<0.001^{\dagger}$ & $0.518^{\dagger}$ \\
\hline $\begin{array}{l}\text { Received prior visual acuity } \\
\text { screening, } n(\%)\end{array}$ & $174(3.00 \%)$ & $0(0.00)$ & $0(0.00)$ & $174(8.00 \%)$ & $\mu^{* *}$ & $\rho^{* *}$ & $\rho^{* *}$ \\
\hline \multicolumn{8}{|l|}{ School characteristics } \\
\hline Public school, $n(\%)$ & $17(26.2 \%)$ & $5(16.7 \%)$ & $6(40.0 \%)$ & $6(30.0 \%)$ & $0.676^{*}$ & $0.870^{*}$ & $0.558^{*}$ \\
\hline Student-teacher ratio (SD) & $22.7(8.81)$ & $18.6(7.64)$ & $24.7(9.57)$ & $24.3(7.85)$ & $0.071^{\dagger}$ & $0.041^{\dagger}$ & $0.896^{\dagger}$ \\
\hline $\begin{array}{l}\text { Distance between school } \\
\text { and county seat }(\mathrm{km}) \\
\text { (Median, IQR) }\end{array}$ & $20.0(10.0-30.0)$ & $10.0(2.00-25.0)$ & $30.0(15.0-45.0)$ & $15.0(10.0-30.0)$ & $0.014^{\dagger}$ & $0.402^{\dagger}$ & $0.044^{\dagger}$ \\
\hline
\end{tabular}

$\mathrm{IQR}=$ Inter quartile range, $\mathrm{SD}=$ Standard deviation.

${ }^{*}$ Logistic regression was used for the comparison adjusting for cluster effect within school.

$\dagger$ Linear regression was used for the comparison adjusting for cluster effect within school.

* The comparison did not perform due to no student received prior visual acuity screening in two of three study groups. 
Table 2. Accuracy of visual acuity screening by local optometrists, teachers and volunteers compared with tertiary optometrists ("gold standard")

\begin{tabular}{|c|c|c|c|c|}
\hline & $\begin{array}{l}\text { Overall } \\
(95 \% \mathrm{CI})\end{array}$ & $\begin{array}{c}\text { Local } \\
\text { optometrist } \\
\text { screening model } \\
(95 \% \mathrm{CI})\end{array}$ & $\begin{array}{c}\text { Teacher } \\
\text { screening model } \\
(95 \% \mathrm{CI})\end{array}$ & $\begin{array}{c}\text { Volunteer } \\
\text { screening } \\
\text { model } \\
(95 \% \mathrm{CI})\end{array}$ \\
\hline Sensitivity & $\begin{array}{c}80.0 \% \\
(61.4 \%, 92.3 \%)\end{array}$ & $\begin{array}{c}76.9 \% \\
(46.2 \%, 95.0 \%)\end{array}$ & $\begin{array}{c}77.8 \% \\
(40.0 \%, 97.2 \%)\end{array}$ & $\begin{array}{c}87.5 \% \\
(47.3 \%, 99.7 \%)\end{array}$ \\
\hline Specificity & $\begin{array}{c}85.6 \% \\
(77.6 \%, 91.5 \%)\end{array}$ & $\begin{array}{c}84.9 \% \\
(68.1 \%, 94.9 \%)\end{array}$ & $\begin{array}{c}86.7 \% \\
(69.3 \%, 96.2 \%)\end{array}$ & $\begin{array}{c}85.4 \% \\
(72.2 \%, 93.9 \%)\end{array}$ \\
\hline $\begin{array}{l}\text { Standardized } \\
\text { PPV }\end{array}$ & $\begin{array}{c}85.1 \% \\
(73.3 \%, 91.6 \%)\end{array}$ & $\begin{array}{c}83.7 \% \\
(59.2 \%, 94.9 \%)\end{array}$ & $\begin{array}{c}85.7 \% \\
(56.6 \%, 96.2 \%)\end{array}$ & $\begin{array}{c}85.4 \% \\
(63.0 \%, 94.2 \%)\end{array}$ \\
\hline
\end{tabular}

PPV: Positive Predictive Value 
Table 3. Incremental cost-effectiveness ratios of different vision screening models*

\begin{tabular}{|c|c|c|c|}
\hline & $\begin{array}{c}\text { Teacher } \\
\text { screening } \\
\text { model }\end{array}$ & $\begin{array}{l}\text { Local optometrist } \\
\text { screening model }\end{array}$ & $\begin{array}{c}\text { Volunteer } \\
\text { screening } \\
\text { model }\end{array}$ \\
\hline Cost per screening $(\mathrm{C})$ & 0.61 & 1.15 & 1.2 \\
\hline Proportion of vision disorders $(\%)(E)$ & 1.6 & 2 & 2.2 \\
\hline Incremental cost $(\Delta \mathrm{C})$ & 0.61 & 0.54 & 0.05 \\
\hline $\begin{array}{l}\text { Incremental proportion of vision } \\
\text { disorders }(\%)(\Delta E)\end{array}$ & 1.6 & 0.4 & 0.2 \\
\hline $\begin{array}{l}\text { Incremental cost-effectiveness ratio } \\
(\Delta \mathrm{C} / \Delta \mathrm{E})^{\dagger}\end{array}$ & 0.38 & 1.35 & 0.25 \\
\hline \multicolumn{4}{|c|}{$\begin{array}{l}\Delta \mathrm{C}=\text { Incremental cost; } \Delta \mathrm{E}=\text { Incremental proportion of vision disorders. } \\
* \text { All costs were reported in } 2015 \mathrm{US} \text { dollars ( } 1 \mathrm{RMB}=0.15 \mathrm{USD}) \text {. } \\
{ }^{\dagger} \text { Incremental cost-effectiveness ratio (ICER) refers to the additional cost of a specific } \\
\text { screening model, divided by the additional true-positive case of failed vision } \\
\text { screening detection, compared with the next less-expensive model. A higher ICER } \\
\text { indicates a worse-performing model. The three models were ranked according to their } \\
\text { effectiveness. The ICERs were calculated using the teacher screening model as the } \\
\text { baseline because its effectiveness was the highest. The local optometrist screening } \\
\text { model was excluded since its ICER was the highest. }\end{array}$} \\
\hline
\end{tabular}


Table 4. Exclusion of more costly and less effective vision screening models

\begin{tabular}{|c|c|c|}
\hline & $\begin{array}{c}\text { Teacher } \\
\text { screening model* }\end{array}$ & $\begin{array}{c}\text { Volunteer } \\
\text { screening model* }\end{array}$ \\
\hline Cost per screening $(\mathrm{C})$ & 0.61 & 1.2 \\
\hline Proportion of vision disorders $(\%)(E)$ & 1.6 & 2.2 \\
\hline Incremental cost $(\Delta \mathrm{C})$ & 0.61 & 0.59 \\
\hline $\begin{array}{l}\text { Incremental proportion of vision } \\
\text { disorders }(\%)(\Delta \mathrm{E})\end{array}$ & 1.6 & 0.6 \\
\hline $\begin{array}{l}\text { Incremental cost-effectiveness ratio } \\
(\Delta \mathrm{C} / \Delta \mathrm{E})\end{array}$ & 0.38 & 0.98 \\
\hline \multicolumn{3}{|c|}{$\begin{array}{l}\Delta \mathrm{C}=\text { Incremental cost; } \Delta \mathrm{E}=\text { Incremental proportion of vision disorders. } \\
* \text { The two screening models were ranked according to their effectiveness. The } \\
\text { incremental cost-effectiveness ratios (ICERs) were calculated using the teacher } \\
\text { screening model as the baseline because its effectiveness was higher. The volunteer } \\
\text { screening model was excluded since its ICER was the highest. }\end{array}$} \\
\hline
\end{tabular}




\section{Appendix}

Table A1. Costs of three different screening models*

\begin{tabular}{|c|c|c|c|}
\hline & $\begin{array}{c}\text { Teacher } \\
\text { screening } \\
\text { model }\end{array}$ & $\begin{array}{c}\text { Local } \\
\text { optometrist } \\
\text { screening model }\end{array}$ & $\begin{array}{c}\text { Volunteer } \\
\text { screening } \\
\text { model }\end{array}$ \\
\hline Training costs $\dagger$ & 930 & 480 & 518 \\
\hline Screening costs $\ddagger$ & 196 & 1,591 & 1,988 \\
\hline Total costs ${ }^{\S}$ & 1,126 & 2,071 & 2,505 \\
\hline Total number of children screened & 1,835 & 1,718 & 2,183 \\
\hline Cost per screening & 0.61 & 1.20 & 1.15 \\
\hline
\end{tabular}

* All costs were reported in US dollars based on the official currency exchange rate in $2015(1$ Renminbi $=$ US\$0.15).

$\uparrow$ Training costs included transportation cost, accommodation, and remuneration for local optometrists and teachers for attending the training.

+ Screening costs included transportation cost, accommodation for volunteers, and subsidies for teachers and local optometrists. we compensate teachers on an hourly basis what same as local optometrists (US\$26 per day, which is US\$3.29 per hour). Specifically, it took 1.5 minutes to screen a child by a teacher, and the total time for all screening was about 46 hours in the teacher screening model. The total salary to pay for the teacher screening model was US\$151.

$\S$ The fixed costs, such as tool kits and training materials, were excluded. 